\title{
Saturation Results for the Truncated Max-Product Sampling Operators Based on Sinc and Fejér-Type Kernels
}

\author{
Lucian Coroianu and Sorin G. Gal \\ Department of Mathematics and Computer Science, \\ The University of Oradea \\ Universitatii 1, 410087 \\ Oradea, Romania \\ lcoroianu@uoradea.ro and galso@uoradea.ro
}

\begin{abstract}
In this paper we obtain the saturation order, a strong localization result and local saturation results in the approximation of continuous positive functions by the truncated max-product sampling operators based on sinc (Wittaker-type) and Fejér-type kernels. The localization-type results obtained present important potential applications in signal theory.
\end{abstract}

Key words and phrases : Max-product truncated sampling operators, signal theory, sinc (Whitaker-type) kernel, Fejér-type kernel, saturation order, local inverse saturation result, localization result, local direct saturation result, Lipschitz function on subintervals.

2000 AMS Mathematics Subject Classification - Primary 94A20, 94A12, 41A36, 41A40 ; Secondary 41A27, 41A20.

\section{Introduction}

The sinc-approximation operators were first introduced and studied in [15], [5] and [20] under the name of cardinal function and of truncated cardinal function. Later on, these linear approximation operators were intensively studied in e.g. [1]-[3], [6]-[9], [13], [14], [16], [17], [18], [19] (see also the references cited there).

Based on the Open Problem 5.5.4, pp. 324-326 in [12], in a series of recent papers submitted for publication we have introduced and studied the so-called max-product operators attached to the Bernstein polynomials and to other linear Bernstein-type operators, like those of Favard-Szász-Mirakjan operators (truncated and nontruncated case), Baskakov operators (truncated and nontruncated case), Meyer-König and Zeller operators and Bleimann-Butzer-Hahn operators.

This idea applied, for example, to the linear Bernstein operators $B_{n}(f)(x)=$ $\sum_{k=0}^{n} p_{n, k}(x) f(k / n)$, where $p_{n, k}(x)=\left(\begin{array}{l}n \\ k\end{array}\right) x^{k}(1-x)^{n-k}$ and $f$ is positive, works 
as follows. Writing in the equivalent form $B_{n}(f)(x)=\frac{\sum_{k=0}^{n} p_{n, k}(x) f(k / n)}{\sum_{k=0}^{n} p_{n, k}(x)}$ and then replacing the sum operator $\Sigma$ by the maximum operator $\bigvee$, one obtains the nonlinear Bernstein operator of max-product kind

$$
B_{n}^{(M)}(f)(x)=\frac{\bigvee_{k=0}^{n} p_{n, k}(x) f\left(\frac{k}{n}\right)}{\bigvee_{k=0}^{n} p_{n, k}(x)}, x \in[0,1], n \in \mathbb{N},
$$

where the notation $\bigvee_{k=0}^{n} p_{n, k}(x)$ means $\max \left\{p_{n, k}(x) ; k \in\{0, \ldots, n\}\right\}$ and similarly for the numerator.

For this nonlinear max-product operator nice approximation and shape preserving properties were found in e.g. [4].

In the recent paper [10], Theorem 3.2 (iv), applying this idea to the classical Whittaker's cardinal series, we have obtained a Jackson-type estimate in terms of $\omega_{1}(f ; 1 / W)_{\mathbb{R}}$, in approximation of a continuous, positive and bounded function $f$ on $\mathbb{R}$, by the nonlinear max-product Whittaker sampling operator given by

$$
S_{W, \varphi}^{(M)}(f)(t)=\frac{\bigvee_{k=-\infty}^{\infty} \varphi(W t-k) f\left(\frac{k}{W}\right)}{\bigvee_{k=-\infty}^{\infty} \varphi(W t-k)}, t \in \mathbb{R}, W>0,
$$

where $\varphi$ is the sinc kernel given by the formula $\varphi(t)=\operatorname{sinc}(t)=\frac{\sin (\pi t)}{\pi t}$. Also, replacing above $\varphi(t)=\frac{1}{2} \cdot \operatorname{sinc}^{2}(t / 2)$ (the Fejér-type kernel), in [11], Theorem 2.4 , we get the same Jackson-type order $\omega_{1}(f ; 1 / W)_{\mathbb{R}}$ in the approximation of a continuous, positive and bounded function $f$ on $\mathbb{R}$. In order to be of interest, only functions $f$ that are uniformly continuous on $\mathbb{R}$ must be considered, because they have the property that $\omega_{1}(f ; t)_{\mathbb{R}} \rightarrow 0$ as $t \rightarrow 0$.

It is worth noting that comparing with the classical linear sampling operators to which they were attached, when for $f$ bounded and Lipschitz of order $\alpha \in(0,1)$ the order of approximation is $\frac{\log (n)}{n^{\alpha}}$ (see Butzer [6] or Butzer-Stens $[9]$ ), these two kinds of nonlinear max-product sampling operators give the approximation order $\frac{1}{n^{\alpha}}$, which is an essential improvement.

Going further and applying the max-product idea to the truncated Whittaker series defined by (see e.g. Borel [5], Whittaker [20])

$$
W_{n}(f)(x)=\sum_{k=0}^{n} \frac{\sin (n x-k \pi)}{n x-k \pi} \cdot f\left(\frac{k \pi}{n}\right), x \in[0, \pi], n \in \mathbb{N},
$$

and to the truncated sampling series based on the Fejér's kernel and defined by

$$
T_{n}(f)(x)=\sum_{k=0}^{n} \frac{\sin ^{2}(n x-k \pi)}{(n x-k \pi)^{2}} \cdot f\left(\frac{k \pi}{n}\right), x \in[0, \pi], n \in \mathbb{N},
$$


in the very recent paper [11], we introduced the truncated max-product Whittaker operator given by

$$
W_{n}^{(M)}(f)(x)=\frac{\bigvee_{k=0}^{n} \frac{\sin (n x-k \pi)}{n x-k \pi} \cdot f\left(\frac{k \pi}{n}\right)}{\bigvee_{k=0}^{n} \frac{\sin (n x-k \pi)}{n x-k \pi}}, x \in[0, \pi], n \in \mathbb{N},
$$

and the truncated max-product operator based on the Fejér-type kernel, given by

$$
T_{n}^{(M)}(f)(x)=\frac{\bigvee_{k=0}^{n} \frac{\sin ^{2}(n x-k \pi)}{(n x-k \pi)^{2}} \cdot f\left(\frac{k \pi}{n}\right)}{\bigvee_{k=0}^{n} \frac{\sin ^{2}(n x-k \pi)}{(n x-k \pi)^{2}}}, x \in[0, \pi], n \in \mathbb{N},
$$

for which the Jackson-type order $\omega_{1}(f ; 1 / n)_{[0, \pi]}$ in the approximation of $f$ was obtained. It is worth noting that the situation is completely different in the case of approximation by their linear counterparts $W_{n}$ and $T_{n}$, when for the uniform convergence in compacts subintervals in $(0, \pi)$ and pointwise convergence in

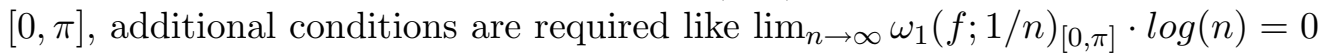
(see Trynin [19]), contrariwise divergence properties may appear (see Sklyarov [16]).

Also, the interpolation property is preserved by the operators $W_{n}^{(M)}$ and $T_{n}^{(M)}$, i.e. we have $W_{n}^{(M)}(f)(j \pi / n)=T_{n}^{(M)}(f)(j \pi / n)=f(j \pi / n), j \in\{0,1, \ldots, n\}$ (see [11]).

Due to the better approximation properties, it is natural to look for a complete characterization of the approximation properties of the nonlinear maxproduct sampling operators $T_{n}^{(M)}$ and $W_{n}^{(M)}$. In this sense, the goal of the present paper is to obtain for them the saturation order, a strong localization result and local inverse and local direct saturation results.

It is worth noting, for example, that the strong localization results in the Theorems 5.1 and 6.1 and the local shape preserving properties in the Corollaries 5.3 and 6.4, clearly show the important advantage that may have the truncated max-product operators $T_{n}^{(M)}(f)$ and $W_{n}^{(M)}(f)$ in the local representation with great accuracy of a continuous non-smooth signal $f$, if we compare them with the linear sampling operators $T_{n}(f)$ and $W_{n}(f)$.

The plan of the paper goes as follows : in Section 2 we deal with the saturation order for $T_{n}^{(M)}$, Section 3 deals with the saturation order for $W_{n}^{(M)}$, in Section 4 a local inverse saturation result for $T_{n}^{(M)}$ is presented, Section 5 contains a localization result and a local direct saturation result for $T_{n}^{(M)}$, while in Section 6 a localization result and local inverse and local direct saturation results for $W_{n}^{(M)}$ are presented.

Everywhere, by convention we take $\sin (0) / 0=1$, which means that for every $x=k \pi / n, k \in\{0,1, \ldots, n\}$, we take $\frac{\sin (n x-k \pi)}{n x-k \pi}=1$. 


\section{The Saturation Order for the $T_{n}^{(M)}$ Operator}

Firstly, we need some auxiliary results.

Lemma 2.1 Denoting $s_{n, k}(x)=\frac{\sin ^{2}(n x-\pi k)}{(n x-\pi k)^{2}}$, for any $j \in\{0, \ldots, n\}$ we have

$$
\bigvee_{k=0}^{n} s_{n, k}(x)=s_{n, j}(x), \quad x \in[(j-1 / 2) \pi / n,(j+1 / 2) \pi / n] \bigcap[0, \pi]
$$

Proof. See the proof of Lemma 4.3 in [11].

Lemma 2.2. For any function $f:[0, \pi] \rightarrow \mathbb{R}_{+}$, where $\mathbb{R}_{+}=\{x \in \mathbb{R} ; x \geq$ $0\}$, and for all $n \in \mathbb{N}, n \geq 1$, and $j \in\{0,1, \ldots, n\}, j \leq n / 2$, we have:

(i)

$$
T_{n}^{(M)}(f)(j \pi /(n+1)) \geq f(j \pi / n) ;
$$

(ii)

$$
T_{n+1}^{(M)}(f)(j \pi / n) \geq f(j \pi /(n+1)) .
$$

Proof. (i) Firstly, by Lemma 2.1 we observe that for $x \in\left[\frac{[(j-1)+1 / 2] \pi}{n}, \frac{j \pi}{n}\right]$ we have $\bigvee_{k=0}^{n} s_{n, k}(x)=s_{n, j}(x)$. Now, if $j \leq n / 2$ then it is easy to check that $x:=$ $j \pi /(n+1) \in\left[\frac{[(j-1)+1 / 2] \pi}{n}, \frac{j \pi}{n}\right]$ which implies $\bigvee_{k=0}^{n} s_{n, k}(j \pi /(n+1))=s_{n, j}(j \pi /(n+$ 1)). This implies that

$$
\begin{aligned}
T_{n}^{(M)}(f)(j \pi /(n+1)) & =\frac{\bigvee_{k=0}^{n} s_{n, k}(j \pi /(n+1)) f\left(\frac{k \pi}{n}\right)}{s_{n, j}(j \pi /(n+1))} \\
& \geq \frac{s_{n, j}(j \pi /(n+1)) f\left(\frac{j \pi}{n}\right)}{s_{n, j}(j \pi /(n+1))}=f\left(\frac{j \pi}{n}\right) .
\end{aligned}
$$

(ii) Since $j \leq n / 2$, one can easily prove that $j \pi / n \in\left[\frac{j \pi}{n+1}, \frac{(j+1 / 2) \pi}{n+1}\right]$. Therefore, by Lemma 2.1 we obtain $\bigvee_{k=0}^{n+1} s_{n+1, k}(j \pi / n)=s_{n+1, j}(j \pi / n)$. This implies that

$$
\begin{aligned}
T_{n+1}^{(M)}(f)(j \pi / n) & =\frac{\bigvee_{k=0}^{n+1} s_{n+1, k}(j \pi / n) f\left(\frac{k \pi}{n+1}\right)}{s_{n+1, j}(j \pi / n)} \geq \frac{s_{n+1, j}(j \pi / n) f\left(\frac{j \pi}{n+1}\right)}{s_{n+1, j}(j \pi / n)} \\
& =f\left(\frac{j \pi}{n+1}\right) .
\end{aligned}
$$


Since $j \geq n / 2$ easily implies that $(j+1) \pi /(n+1) \in[j \pi / n,(j+1 / 2) \pi / n]$ and $j \pi / n \in[(j+1 / 2) \pi /(n+1),(j+1) \pi /(n+1)]$, by similar reasonings with those for Lemma 2.2, we also get the following.

Lemma 2.3. For any function $f:[0, \pi] \rightarrow \mathbb{R}_{+}$and for all $n \in \mathbb{N}, n \geq 1$, and $j \in\{0,1, \ldots, n\}, j \geq n / 2$, we have:

(i)

$$
T_{n}^{(M)}(f)((j+1) \pi /(n+1)) \geq f(j \pi / n) ;
$$

(ii)

$$
T_{n+1}^{(M)}(f)(j \pi / n) \geq f((j+1) \pi /(n+1)) .
$$

We are now in position to determine the saturation order and the associated special class of functions for the truncated max-product operator $T_{n}^{(M)}$.

Theorem 2.4. Denote $C_{+}[0, \pi]=\left\{f:[0, \pi] \rightarrow \mathbb{R}_{+} ; f\right.$ continuous on $\left.[0, \pi]\right\}$ and $\|f\|=\sup \{|f(x)| ; x \in[0, \pi]\}$. Then for the max-product $T_{n}^{(M)}$ operator, the saturation order in $C_{+}[0, \pi]$ is $\frac{1}{n}$, that is $\left\|T_{n}^{(M)}(f)-f\right\|=o(1 / n)$, implies that $f$ is a constant function on $[0, \pi]$.

Proof. By hypothesis, there exists $a_{n} \in \mathbb{R}, n \in \mathbb{N}$ with the property $a_{n} \searrow 0$ as $n \rightarrow+\infty$, such that

$$
\left|T_{n}^{(M)}(f)(x)-f(x)\right| \leq \frac{a_{n}}{n}, \text { for all } x \in[0, \pi] \text { and } n \in \mathbb{N} .
$$

Let us choose arbitrary $\varepsilon>0$. Since $a_{n} \searrow 0$ as $n \rightarrow+\infty$, it follows that there exists $n_{0} \in \mathbb{N}$ such that $a_{n}<\varepsilon$ for all $n \in \mathbb{N}, n \geq n_{0}$. Noting the above relation we get

$$
\left|T_{n}^{(M)}(f)(x)-f(x)\right| \leq \frac{\varepsilon}{n}, \text { for all } x \in[0, \pi] \text { and } n \in \mathbb{N}, n \geq n_{0} .
$$

Then, from the uniform continuity of $f$ it results the existence of $n_{1} \in \mathbb{N}$ such that

$$
|f(x)-f(y)| \leq \varepsilon \text { for all } x, y \in[0, \pi] \text { and } n \in \mathbb{N},|x-y| \leq \pi / n, n \geq n_{1} .
$$

We will obtain the desired conclusion by following the following steps: (A) we prove that $f$ is constant on any interval $[a, b]$ with $0<a<b<\pi / 2$; (B) we prove that $f$ is constant on any interval $[a, b]$ with $\pi / 2<a<b<1$. Indeed, if (A) holds then thanks to the continuity of $f$ we easily obtain that $f$ is constant on $[0, \pi / 2]$. Similarly, if (B) holds then we obtain that $f$ is constant on $[\pi / 2,1]$. Then, from the continuity of $f$ it easily follows that $f$ is constant on $[0, \pi]$. So, we start by proving that (A) and (B) hold.

(A) Let us choose arbitrary $a, b \in \mathbb{R}$ such that $0<a<b<\pi / 2$. Further one, let $x_{0}$ and $y_{0}$ be the points where $f$ attaints its minimum and maximum respectively on the interval $[a, b]$. Without any loss of generality we may suppose 
that $x_{0} \neq y_{0}$ (contrariwise it follows that $f$ is constant on $[a, b]$ and there is nothing to prove). We have two subcases: $\left.\mathrm{A}_{1}\right) x_{0}<y_{0}$ and $\left.\mathrm{A}_{2}\right) x_{0}>y_{0}$.

Subcase $\left.\mathrm{A}_{1}\right)$ Let us choose arbitrary $n \in \mathbb{N}$ with $n>\max \left\{n_{0}, n_{1}, 2 \pi /\left(y_{0}-\right.\right.$ $\left.\left.x_{0}\right)\right\}$. By relation (1) it follows that

$$
T_{n}^{(M)}(f)(j \pi /(n+1))-f(j \pi /(n+1)) \leq \frac{\varepsilon}{n} \text { for all } j \in\{0,1, \ldots, n\} .
$$

Moreover, combining the inequality in Lemma 2.2 (i) with the above inequality, we get

$$
f(j \pi / n)-f(j \pi /(n+1)) \leq \frac{\varepsilon}{n} \text { for all } j \in\{0,1, \ldots, n\}, j \leq n / 2 .
$$

Further one, let us choose $j_{1} \in\{0,1, \ldots, n-1\}$ such that $j_{1} \pi / n \leq y_{0} \leq\left(j_{1}+1\right) \pi / n$ and $x_{0} \leq j_{1} \pi / n$. Note that there exists such an index $j_{1}$, because the previous inequalities are equivalent to $\frac{n y_{0}}{\pi}-1 \leq j_{1} \leq \frac{n y_{0}}{\pi}, \frac{n x_{0}}{\pi} \leq j_{1} \leq \frac{n y_{0}}{\pi}$, while the condition $n>2 \pi /\left(y_{0}-x_{0}\right)$ is equivalent to the condition $\frac{n y_{0}}{\pi}-\frac{n x_{0}}{\pi}>2$.

Also, from $j_{1} \pi / n \leq y_{0} \leq b<\pi / 2$ it easily follows that $j_{1} \leq n / 2$.

As a first consequence, from the relation (2) we obtain

$$
\left|f\left(j_{1} \pi / n\right)-f\left(y_{0}\right)\right|<\varepsilon .
$$

Then, since $\lim _{l \rightarrow \infty} \frac{j_{1} \pi}{n+l}=0$, by $x_{0}>0$ and $x_{0} \leq j_{1} \pi / n$ it follows that there exists $l_{0} \in \mathbb{N}$ such that $\frac{j_{1} \pi}{n+l_{0}+1} \leq x_{0} \leq \frac{j_{1} \pi}{n+l_{0}}$.

It is worth noting here that indeed, the above $l_{0}$ cannot be equal to 0 , because if we would have $l_{0}=0$, then we would obtain $j_{1} \pi /(n+1) \leq x_{0}<y_{0} \leq$ $\left(j_{1}+1\right) \pi / n \leq\left(j_{1}+2\right) \pi /(n+1)$, which would imply $y_{0}-x_{0} \leq 2 \pi /(n+1)<2 \pi / n$, in contradiction with the supposition that $n>2 \pi /\left(y_{0}-x_{0}\right)$.

The inequality $\frac{j_{1} \pi}{n+l_{0}+1} \leq x_{0} \leq \frac{j_{1} \pi}{n+l_{0}}$ and (2) also implies that

$$
\mid f\left(\left(j_{1} \pi /\left(n+l_{0}\right)\right)-f\left(x_{0}\right) \mid<\varepsilon .\right.
$$

Since $j_{1} \leq n / 2$, applying successively relation (3) we obtain

$$
\begin{aligned}
f\left(j_{1} \pi / n\right)-f\left(j_{1} \pi /(n+1)\right) & \leq \frac{\varepsilon}{n}, \\
f\left(j_{1} \pi /(n+1)\right)-f\left(j_{1} \pi /(n+2)\right) & \leq \frac{\varepsilon}{n+1}, \\
f\left(j_{1} \pi /\left(n+l_{0}-1\right)\right)-f\left(j_{1} \pi /\left(n+l_{0}\right)\right) & \leq \frac{\varepsilon}{n+l_{0}-1} .
\end{aligned}
$$

Taking the sum of all these inequalities we get

$$
\begin{aligned}
f\left(j_{1} \pi / n\right)-f\left(j_{1} \pi /\left(n+l_{0}\right)\right) & \leq \frac{\varepsilon}{n}+\frac{\varepsilon}{n+1}+\ldots+\frac{\varepsilon}{n+l_{0}-1} \\
& \leq \frac{l_{0} \varepsilon}{n} .
\end{aligned}
$$


Then, by relations (4)-(5) we obtain

$$
\begin{aligned}
& f\left(y_{0}\right)-f\left(x_{0}\right) \\
= & \left(f\left(y_{0}\right)-f\left(j_{1} \pi / n\right)\right)+\left(f\left(j_{1} \pi / n\right)-f\left(j_{1} \pi /\left(n+l_{0}\right)\right)\right) \\
& +\left(f\left(j_{1} \pi /\left(n+l_{0}\right)\right)-f\left(x_{0}\right)\right) \\
\leq & \left|f\left(y_{0}\right)-f\left(j_{1} \pi / n\right)\right|+f\left(j_{1} \pi / n\right)-f\left(j_{1} \pi /\left(n+l_{0}\right)\right) \\
& +\left|f\left(j_{1} \pi /\left(n+l_{0}\right)\right)-f\left(x_{0}\right)\right| \\
\leq & 2 \varepsilon+\frac{l_{0} \varepsilon}{n}
\end{aligned}
$$

and since $0 \leq f\left(y_{0}\right)-f\left(x_{0}\right)$, we obtain

$$
0 \leq f\left(y_{0}\right)-f\left(x_{0}\right) \leq 2 \varepsilon+\frac{l_{0} \varepsilon}{n} .
$$

On the other hand, since $0<x_{0} \leq j_{1} \pi /\left(n+l_{0}\right)$, after some simple calculations we get (note that $j_{1} \leq n / 2$ )

$$
l_{0} \leq j_{1} \pi / x_{0}-n \leq n\left(\pi /\left(2 x_{0}\right)-1\right)
$$

Using this information in relation (6) we obtain

$$
0 \leq f\left(y_{0}\right)-f\left(x_{0}\right) \leq \varepsilon\left(2+\pi /\left(2 x_{0}\right)-1\right)
$$

where $\varepsilon>0$ was chosen arbitrary. Therefore, passing to the limit for $\varepsilon \searrow 0$ in the previous inequality, we obtain $f\left(x_{0}\right)=f\left(y_{0}\right)$ (here, it is important that $\left.x_{0}>0\right)$. Since on the interval $[a, b]$ the maximum value and the minimum value of the function $f$ coincide, we obtain that $f$ is a constant function on the interval $[a, b]$ and hence we obtained the desired conclusion for this case.

The proof of Subcase $A_{2}$ ) is similar to that in the case of Subcase $A_{1}$ ), which proves the Case $(\mathrm{A})$.

(B) Let us choose arbitrary $a, b \in \mathbb{R}$ such that $\pi / 2<a<b<\pi$ and further one, let $x_{0}$ and $y_{0}$ be the points where $f$ attaints its minimum and maximum respectively on the interval $[a, b]$. Again we have two subcases $\left.B_{1}\right) x_{0}<y_{0}$ and $\left.B_{2}\right) x_{0}>y_{0}$, which by similar reasonings with those in the Case (A) implies that $(B)$ holds.

Now, by the discussion just before the beginning of the case (A), we conclude that $f$ is constant on the whole interval $[0, \pi]$.

Remark. Because it is easy to check that $T_{n}^{(M)}$ reproduces the constant functions in $C_{+}[0, \pi]$, it follows that the saturation class in $C_{+}[0, \pi]$ for $T_{n}^{(M)}$ is exactly the class of constant functions. 


\section{The Saturation Order for the $W_{n}^{(M)}$ Operator}

Firstly, we need some auxiliary results.

Lemma 3.1 Denoting now $s_{n, k}(x)=\frac{\sin (n x-\pi k)}{n x-\pi k}$, for any $j \in\{0, \ldots, n\}$ we have

$$
\bigvee_{k=0}^{n} s_{n, k}(x)=s_{n, j}(x), \quad x \in[(j-1 / 2) \pi / n,(j+1 / 2) \pi / n] \bigcap[0, \pi]
$$

Proof. See the proof of Lemma 3.3 in [11].

Lemma 3.2. For any function $f:[0, \pi] \rightarrow \mathbb{R}_{+}$and for all $n \in \mathbb{N}, n \geq 1$, and $j \in\{0,1, \ldots, n\}, j \leq n / 2$, we have:

(i)

$$
W_{n}^{(M)}(f)(j \pi /(n+1)) \geq f(j \pi / n) ;
$$

(ii)

$$
W_{n+1}^{(M)}(f)(j \pi / n) \geq f(j \pi /(n+1)) .
$$

Proof. The proof is identical with the proof of Lemma 2.2 .

Lemma 3.3. For any function $f:[0, \pi] \rightarrow \mathbb{R}_{+}$and for all $n \in \mathbb{N}, n \geq 1$, and $j \in\{0,1, \ldots, n\}, j \geq n / 2$, we have:

$$
W_{n}^{(M)}(f)((j+1) \pi /(n+1)) \geq f(j \pi / n) ;
$$

(ii)

$$
W_{n+1}^{(M)}(f)(j \pi / n) \geq f((j+1) \pi /(n+1)) .
$$

Proof. The proof is identical with the proof of Lemma 2.3.

Since Theorem 2.4 is a direct consequence of the Lemmas 2.1-2.3 and in its proof does not intervene at all the form of the kernel $s_{n, k}(x)$, as a consequence of the Lemmas 3.1-3.3, analogously we can prove the following saturation result for the truncated max-product operator $W_{n}^{(M)}$.

Theorem 3.4. For the max-product $W_{n}^{(M)}$ operator, the saturation order in $C_{+}[0, \pi]$ is $\frac{1}{n}$, that is $\left\|W_{n}^{(M)}(f)-f\right\|=o(1 / n)$, implies that $f$ is a constant function on $[0, \pi]$.

Remark. Because it is easy to see that $W_{n}^{(M)}$ reproduces the constant functions in $C_{+}[0, \pi]$, it follows that the saturation class for $W_{n}^{(M)}$ in $C_{+}[0, \pi]$ is exactly the class of constant functions.

\section{Local Inverse Saturation Result for the $T_{n}^{(M)}$ Oper- ator}

In this section we present the following local inverse saturation result for the operator $T_{n}^{(M)}$. 
Theorem 4.1. Let $f:[0, \pi] \rightarrow \mathbb{R}_{+}$and $0<\alpha<\beta<\pi$ be such that $f$ is continuous on $[\alpha, \beta]$. If there exists a constant $M>0$ (independent of $n$ but depending on $f, \alpha$ and $\beta$ ) such that

$$
\left\|T_{n}^{(M)}(f)-f\right\|_{[\alpha, \beta]} \leq M / n, \text { for all } n \in \mathbb{N},
$$

then $\left.f\right|_{[\alpha, \beta]} \in \operatorname{Lip}[\alpha, \beta]$, that is $f$ is a Lipschitz function on $[\alpha, \beta]$. Here $\|f\|_{[\alpha, \beta]}=\sup \{|f(x)| ; x \in[\alpha, \beta]\}$ and

$$
\operatorname{Lip}[\alpha, \beta]=\{g:[\alpha, \beta] \rightarrow \mathbb{R} ;|g(x)-g(y)| \leq C|x-y|, \text { for all } x, y \in[\alpha, \beta]\} .
$$

The proof of Theorem 4.1 requires the following three lemmas.

Lemma 4.2. Let $f:[0, \pi] \rightarrow \mathbb{R}, n \in \mathbb{N}$ and $0<\alpha<\beta \leq \pi / 2$ be fixed, such that $f$ is continuous on $[\alpha, \beta]$. Also, denote

$$
\begin{gathered}
M_{n}(\alpha, \beta) \\
=\max \left\{\left|f\left(\frac{k \pi}{n}\right)-f\left(\frac{k \pi}{n+1}\right)\right|: k \in\{0, \ldots, n\}, \alpha \leq \frac{k \pi}{n+1} \leq \frac{k \pi}{n} \leq \beta\right\} .
\end{gathered}
$$

Then

$$
\limsup _{n \rightarrow \infty} n \cdot \omega_{1}(f, \pi / n)_{[\alpha, \beta]}=\infty \text { if and only if } \limsup _{n \rightarrow \infty} n \cdot M_{n}(\alpha, \beta)=\infty .
$$

where

$$
\omega_{1}(f, \delta)_{[\alpha, \beta]}=\sup \{|f(x)-f(y)| ; x, y \in[\alpha, \beta],|x-y| \leq \delta\} .
$$

Proof. We prove only the direct implication since the converse one is immediate. Since $f$ is continuous on the interval $[\alpha, \beta]$, it easily follows that for each $n \in \mathbb{N}$, $n \geq 2, \pi / n \leq \beta-\alpha$, there exist $x_{n}, y_{n} \in[\alpha, \beta]$ satisfying $\left|x_{n}-y_{n}\right| \leq \pi / n$ and $\omega_{1}(f, \pi / n)_{[\alpha, \beta]}=\left|f\left(x_{n}\right)-f\left(y_{n}\right)\right|$. Without any loss of generality, we may suppose that $x_{n} \neq y_{n}$ and $x_{n}<y_{n}$, for all $n \in \mathbb{N}$.

Let us consider the sequences $\left(a_{n}\right)_{n \geq 1}$ and $\left(b_{n}\right)_{n \geq 1}, a_{n}=n \omega_{1}(f, \pi / n)_{[\alpha, \beta]}=$ $n\left|f\left(x_{n}\right)-f\left(y_{n}\right)\right|$ and $b_{n}=n \cdot M_{n}(\alpha, \beta)$.

Let us fix $n \in \mathbb{N}$. Since $f$ is uniformly continuous on $[\alpha, \beta]$, it follows that there exists $m \in \mathbb{N}$ such that for all $x, y \in[\alpha, \beta]$ satisfying $|x-y| \leq \pi / m$ we have $|f(x)-f(y)| \leq 1 / n$. In addition, we may choose sufficiently large $m \in \mathbb{N}$ such that $y_{n}-x_{n}>2 \pi / m$, that is $m>2 \pi /\left(y_{n}-x_{n}\right)$.

Since $0<\alpha<y_{n} \leq \beta<\pi / 2$, clearly there exists $j \in\{1, \ldots, m-1\}$ (depending on $m$ and $n)$ such that $j \pi / m \leq y_{n} \leq(j+1) \pi / m$.

Since $\lim _{l \rightarrow \infty} j \pi /(m+l)=0$ and since $x_{n} \geq \alpha>0$, it results the existence of $l_{0} \in \mathbb{N}$ (depending on $j$ and $m$ ) such that $j \pi /\left(m+l_{0}+1\right) \leq x_{n} \leq j \pi /\left(m+l_{0}\right)$.

By the inequalities $x_{n} \leq j \pi /\left(m+l_{0}\right)<j \pi / m \leq y_{n}$, we get

$$
\left|f\left(x_{n}\right)-f\left(y_{n}\right)\right|
$$




$$
\begin{aligned}
\leq & \left|f\left(x_{n}\right)-f\left(j \pi /\left(m+l_{0}\right)\right)\right|+\left|f\left(j \pi /\left(m+l_{0}\right)\right)-f\left(j \pi /\left(m+l_{0}-1\right)\right)\right| \\
& +\ldots+|f(j \pi /(m+1))-f(j \pi / m)|+\left|f(j \pi / m)-f\left(y_{n}\right)\right| \\
\leq & \left|f\left(x_{n}\right)-f\left(j \pi /\left(m+l_{0}\right)\right)\right|+\left|f(j \pi / m)-f\left(y_{n}\right)\right| \\
& +l_{0}|f(j \pi /(m+p))-f(j \pi /(m+p+1))|
\end{aligned}
$$

where $p \in\left\{0,1, \ldots, l_{0}-1\right\}$ is such that

$$
\begin{aligned}
& |f(j \pi /(m+p))-f(j \pi /(m+p+1))| \\
= & \max \left\{|f(j \pi /(m+k))-f(j \pi /(m+k+1))|: k \in\left\{0,1, \ldots, l_{0}-1\right\}\right\} .
\end{aligned}
$$

On the other hand, we observe that $\max \left\{\left|j \pi /\left(m+l_{0}\right)-x_{n}\right|,\left|j \pi / m-y_{n}\right|\right\} \leq$ $\pi / m$, which implies $\left|f\left(x_{n}\right)-f\left(j \pi /\left(m+l_{0}\right)\right)\right| \leq 1 / n$ and $\left|f(j \pi / m)-f\left(y_{n}\right)\right| \leq$ $1 / n$. We thus obtain that

$$
\left|f\left(x_{n}\right)-f\left(y_{n}\right)\right| \leq 2 / n+l_{0}|f(j \pi /(m+p))-f(j \pi /(m+p+1))| .
$$

By the inequalities $x_{n} \leq j \pi /\left(m+l_{0}\right) \leq j \pi / m \leq y_{n}$ we get $j \pi / m-j \pi /(m+$ $\left.l_{0}\right) \leq y_{n}-x_{n} \leq \pi / n$ and this implies $j \pi l_{0} /\left(m\left(m+l_{0}\right)\right) \leq \pi / n$ and then $l_{0} \leq$ $m / j \cdot\left(m+l_{0}\right) / n \leq \pi / \alpha \cdot\left(m+l_{0}\right) / n$. (Here we used that $\left.\alpha \leq x_{n}<j \pi / m\right)$.

Then, by the inequalities $0<\alpha \leq x_{n} \leq A:=j \pi /\left(m+l_{0}\right) \leq B:=j \pi / m \leq$ $y_{n} \leq \beta$ we easily get $B / A \leq \beta / \alpha$, which immediately implies $j \pi /\left(m+l_{0}\right) \geq$ $j \pi / m \cdot \alpha / \beta$. From here we get $m+l_{0} \leq m \beta / \alpha$, that is $l_{0} \leq m(\beta / \alpha-1)$. Replacing this last inequality in the inequality $l_{0} \leq \pi / \alpha \cdot\left(m+l_{0}\right) / n$ just proved above, we conclude that $l_{0} \leq \pi \beta / \alpha^{2} \cdot m / n$.

Replacing now in relation (7) and then multiplying with $n$, we get

$$
\begin{gathered}
n \cdot\left|f\left(x_{n}\right)-f\left(y_{n}\right)\right| \leq 2+\pi \beta / \alpha^{2} \cdot m \cdot|f(j \pi /(m+p))-f(j \pi /(m+p+1))| \\
\leq 2+\pi \beta / \alpha^{2} \cdot(m+p) \cdot|f(j \pi /(m+p))-f(j \pi /(m+p+1))|
\end{gathered}
$$

and clearly this implies that $a_{n} \leq 2+\pi \beta / \alpha^{2} \cdot M_{m+p}(\alpha, \beta)$. Summarizing, for any $n \in \mathbb{N}$ there exist $m+p \in \mathbb{N}$ such that $a_{n} \leq \pi \beta / \alpha^{2} \cdot b_{m+p}+2$. Since $m>$ $2 \pi /\left(y_{n}-x_{n}\right)$ and $y_{n}-x_{n}<\pi / n$, we get $m>2 n$. Therefore, by $\limsup _{n \rightarrow \infty} a_{n}=\infty$, it easily follows that $\limsup _{n \rightarrow \infty} b_{n}=\infty$ and the lemma is proved.

By similar reasoning with those for the Lemma 4.2 , we also get he following

Lemma 4.3. Let $f:[0, \pi] \rightarrow \mathbb{R}, n \in \mathbb{N}$ and $\pi / 2 \leq \alpha<\beta<\pi$ be fixed, such that $f$ is continuous on $[\alpha, \beta]$. Also, denote

$$
\begin{gathered}
P_{n}(\alpha, \beta) \\
=\max \left\{\left|f\left(\frac{k \pi}{n}\right)-f\left(\frac{(k+1) \pi}{n+1}\right)\right|: k \in\{0, \ldots, n\}, \alpha \leq \frac{k \pi}{n} \leq \frac{(k+1) \pi}{n+1} \leq \beta\right\} .
\end{gathered}
$$

Then

$$
\limsup _{n \rightarrow \infty} n \cdot \omega_{1}(f, \pi / n)_{[\alpha, \beta]}=\infty \text { if and only if } \limsup _{n \rightarrow \infty} n \cdot P_{n}(\alpha, \beta)=\infty \text {. }
$$


where

$$
\omega_{1}(f, \delta)_{[\alpha, \beta]}=\sup \{|f(x)-f(y)| ; x, y \in[\alpha, \beta],|x-y| \leq \delta\} .
$$

Lemma 4.4. Let $f:[0, \pi] \rightarrow \mathbb{R}_{+}$and $0<\alpha<\beta<\pi$ be such that $f$ continuous on $[\alpha, \beta]$. If

$$
\limsup _{n \rightarrow \infty} n \cdot \omega_{1}(f, \pi / n)_{[\alpha, \beta]}=\infty,
$$

then

$$
\limsup _{n \rightarrow \infty} n \cdot\left\|T_{n}^{(M)}(f)-f\right\|_{[\alpha, \beta]}=\infty .
$$

Here $\|f\|_{[\alpha, \beta]}=\sup \{|f(x)| ; x \in[\alpha, \beta]\}$.

Proof. If $\alpha<\pi / 2<\beta$ then by the hypothesis it is elementary to prove that either $\limsup _{n \rightarrow \infty} n \cdot \omega_{1}(f, \pi / n)_{[\alpha, \pi / 2]}=\infty$ or $\limsup _{n \rightarrow \infty} n \cdot \omega_{1}(f, \pi / n)_{[\pi / 2, \beta]}=\infty$. Therefore, without any loss of generality we may suppose that we have only two cases: (i) $0<\alpha<\beta \leq \pi / 2$ and (ii) $\pi / 2 \leq \alpha<\beta<\pi$.

Case (i) For fixed $n \in \mathbb{N}$ with $n \geq(\pi+\alpha) /(\beta-\alpha)$, let us choose $k(n) \in$ $\{1, \ldots, n\}$ such that $\alpha \leq \frac{k(n) \pi}{n+1} \leq \frac{k(n) \pi}{n} \leq \beta$ and

$$
M_{n}(\alpha, \beta)=\left|f\left(\frac{k(n) \pi}{n}\right)-f\left(\frac{k(n) \pi}{n+1}\right)\right| .
$$

Note that such an index $k(n)$ exists, because the inequalities $\alpha \leq k(n) \pi /(n+1) \leq$ $k(n) \pi / n \leq \beta$ imply $\alpha(n+1) / \pi \leq k(n) \leq \beta n / \pi$, where $\beta n / \pi-\alpha(n+1) / \pi \geq 1$.

Since $\beta \leq \pi / 2$, it results that $k(n) \leq n / 2$ and hence we can use the conclusion of Lemma 2.2. This means that we have

$$
T_{n}^{(M)}(f)(k(n) \pi /(n+1)) \geq f(k(n) \pi / n)
$$

and

$$
T_{n+1}^{(M)}(f)(k(n) \pi / n) \geq f(k(n) \pi /(n+1)) .
$$

If $f(k(n) \pi / n) \geq f(k(n) \pi /(n+1))$ then

$$
\begin{aligned}
& n \cdot\left(T_{n}^{(M)}(f)(k(n) \pi /(n+1))-f(k(n) \pi /(n+1))\right) \\
\geq & n \cdot(f(k(n) \pi / n)-f(k(n) \pi /(n+1)))=n \cdot M_{n}(\alpha, \beta)
\end{aligned}
$$

and this implies

$$
n \cdot M_{n}(\alpha, \beta) \leq n \cdot\left\|T_{n}^{(M)}(f)-f\right\|_{[\alpha, \beta]} .
$$

If $f(k(n) \pi / n)<f(k(n) \pi /(n+1))$ then

$$
\begin{aligned}
& (n+1) \cdot\left(T_{n+1}^{(M)}(f)(k(n) \pi / n)-f(k(n) \pi / n)\right) \\
\geq \quad & (n+1) \cdot(f(k(n) \pi /(n+1))-f(k(n) \pi / n)) \geq n \cdot M_{n}(\alpha, \beta) .
\end{aligned}
$$


and this implies

$$
n \cdot M_{n}(\alpha, \beta) \leq(n+1) \cdot\left\|T_{n+1}^{(M)}(f)-f\right\|_{[\alpha, \beta]} .
$$

In conclusion, for any $n \in \mathbb{N}$ with $n \geq \pi /(\beta-\alpha)$, we have

$$
n \cdot M_{n}(\alpha, \beta) \leq \max \left\{n \cdot\left\|T_{n}^{(M)}(f)-f\right\|_{[\alpha, \beta]},(n+1) \cdot\left\|T_{n+1}^{(M)}(f)-f\right\|_{[\alpha, \beta]}\right\} .
$$

Since by Lemma 4.3 we have $\lim \sup n \cdot M_{n}(\alpha, \beta)=\infty$, it easily follows now that $\limsup _{n \rightarrow \infty} n \cdot\left\|T_{n}^{(M)}(f)-f\right\|_{[\alpha, \beta]}=\infty$.

The proof of the Case (ii) is absolutely similar, which proves the lemma.

Now, we are in position to prove Theorem 4.1.

Proof of Theorem 4.1. Firstly. it is immediate that $f$ is a Lipschitz function on $[\alpha, \beta]$ if and only if $\limsup _{n \rightarrow \infty} n \cdot \omega_{1}(f, 1 / n)_{[\alpha, \beta]}<\infty$.

Now, by the hypothesis it follows $n \cdot\left\|T_{n}^{(M)}(f)-f\right\|_{[\alpha, \beta]} \leq M$, for all $n \in \mathbb{N}$. Supposing that $f$ is not a Lipschitz function on $[\alpha, \beta]$, by the above considerations it follows that $\lim \sup n \cdot \omega_{1}(f, 1 / n)_{[\alpha, \beta]}=\infty$. But then, by Lemma 4.4 we get

$$
\limsup _{n \rightarrow \infty} n \cdot\left\|T_{n}^{(M)}(f)-f\right\|_{[\alpha, \beta]}=\infty,
$$

which is a contradiction. The theorem is proved.

\section{Localization and Local Direct Saturation Result for the $T_{n}^{(M)}$ Operator}

In this section we obtain a local direct saturation result as a consequence of the following strong localization theorem, of independent importance.

Theorem 5.1. Let $f, g:[0, \pi] \rightarrow \mathbb{R}_{+}$be both bounded on $[0, \pi]$ with strictly positive lower bounds and suppose that there exists $a, b \in[0, \pi], 0<a<b<\pi$ such that $f(x)=g(x)$ for all $x \in[a, b]$. Then for all $c, d \in[a, b]$ satisfying $a<c<d<b$ there exists $\widetilde{n} \in \mathbb{N}$ which depends only on $f, g, a, b, c, d$ such that $T_{n}^{(M)}(f)(x)=T_{n}^{(M)}(g)(x)$ for all $x \in[c, d]$ and $n \in \mathbb{N}, n \geq \widetilde{n}$.

Proof. Let us choose arbitrary $x \in[c, d]$ and for each $n \in \mathbb{N}$ let $j_{x} \in$ $\{0,1, \ldots, n\}$ ( $j_{x}$ depends on $n$ too, but there is no need at all to complicate on the notations) be such that $x \in\left[j_{x} \pi / n,\left(j_{x}+1\right) \pi / n\right]$. Then we know that

$$
T_{n}^{(M)}(f)(x)=\frac{\bigvee_{k=0}^{n} s_{n, k}(x) f\left(\frac{k \pi}{n}\right)}{\bigvee_{k=0}^{n} s_{n, k}(x)},
$$


where for $k \in\{0,1, \ldots, n\}$ we have $s_{n, k}(x)=\frac{\sin ^{2}(n x-k \pi)}{(n x-k \pi)^{2}}$. Since $x \in[c, d] \cap$ $\left[j_{x} \pi / n,\left(j_{x}+1\right) \pi / n\right]$ and since $a<c<d<b$ it is immediate that for $n \geq n_{0}$ where $n_{0}$ is chosen such that $\pi / n_{0}<\min \{c-a, d-b\}$, we obtain $a<j_{x} \pi / n<b$ which gives $n a / \pi<j_{x}<n b / \pi$ for all $n \geq n_{0}$ (indeed, if we would suppose that there exists $n>n_{0}$ which does not satisfy the previous double inequalities, then we would easily get a contradiction).

It is important to notice here that $n_{0}$ does not depend on $x$. From the previous inequality it follows that if $n \geq n_{0}$ then for any $x \in[c, d]$ there exists $\alpha_{x} \in[a, b]$ such that $j_{x}=n \alpha_{x} / \pi$.

In what follows, it will serve to our purpose to use the sequence $\left(a_{n}\right)_{n \geq 1}$, $a_{n}=\sqrt{n}$. For this sequence there exists $n_{1} \in \mathbb{N}$ such that $n a / \pi-a_{n}>0$ for all $n \geq n_{1}$.

Our intention is to prove as an intermediate result, that there exits an absolute constant $N_{0} \in \mathbb{N}$ independent of $x \in[c, d]$ such that for any $n \geq N_{0}$ and $x \in[c, d]$ we have $\bigvee_{k=0}^{n} s_{n, k}(x) f\left(\frac{k \pi}{n}\right)=\bigvee_{k \in I_{n, x}} s_{n, k}(x) f\left(\frac{k \pi}{n}\right)$ where $I_{n, x}=\{k \in$ $\left.\{0,1, \ldots, n\}: j_{x}-a_{n} \leq k \leq j_{x}+a_{n}\right\}$. In order to obtain this conclusion, for $n \geq \max \left\{n_{0}, n_{1}\right\}$ let us choose $k \in\{0,1, \ldots, n\} \backslash I_{n, x}$. We have two cases: i) $k+a_{n}<j_{x}$ and ii) $j_{x}+a_{n}<k$.

Case i) We have two subcases: $\left.\mathrm{i}_{a}\right) x \in\left[j_{x} \pi / n,\left(j_{x}+1 / 2\right) \pi / n\right]$ and $\left.\mathrm{i}_{b}\right) x \in$ $\left[\left(j_{x}+1 / 2\right) \pi / n,\left(j_{x}+1\right) \pi / n\right]$.

Subcase $\left.\mathrm{i}_{a}\right)$ Since $x \in\left[j_{x} \pi / n,\left(j_{x}+1 / 2\right) \pi / n\right]$, we observe that $(n x-$ $\left.j_{x} \pi\right) \in[0, \pi / 2]$ and by the well-known property $\sin (x) \geq 2 x / \pi, x \in[0, \pi / 2]$, it results that $s_{n, j_{x}}(x) \geq 4 / \pi^{2}$. This implies

$$
\begin{aligned}
\frac{s_{n, j_{x}}(x) f\left(\frac{j_{x} \pi}{n}\right)}{s_{n, k}(x) f\left(\frac{k \pi}{n}\right)} & \geq \frac{4 / \pi^{2} \cdot f\left(\frac{j_{x} \pi}{n}\right)}{s_{n, k}(x) f\left(\frac{k \pi}{n}\right)}=\frac{(n x-k \pi)^{2}}{\sin ^{2}(n x-k \pi)} \cdot \frac{4}{\pi^{2}} \cdot \frac{f\left(\frac{j_{x} \pi}{n}\right)}{f\left(\frac{k \pi}{n}\right)} \\
& \geq(n x-k \pi)^{2} \cdot \frac{4}{\pi^{2}} \cdot \frac{f\left(\frac{j_{x} \pi}{n}\right)}{f\left(\frac{k \pi}{n}\right)} .
\end{aligned}
$$

Since $n x \geq j_{x} \pi \geq k \pi$ it follows that

$$
\frac{s_{n, j_{x}}(x) f\left(\frac{j_{x} \pi}{n}\right)}{s_{n, k}(x) f\left(\frac{k \pi}{n}\right)} \geq\left(j_{x} \pi-k \pi\right)^{2} \cdot \frac{4}{\pi^{2}} \cdot \frac{f\left(\frac{j_{x} \pi}{n}\right)}{f\left(\frac{k \pi}{n}\right)} \geq 4 a_{n} \cdot \frac{f\left(\frac{j_{x} \pi}{n}\right)}{f\left(\frac{k \pi}{n}\right)} .
$$

Then, denoting the infimum and the supremum of $f$ on $[0,1]$ with $m_{f}$ and $M_{f}$, respectively (according to the hypotheses they are strictly positive real numbers), we get that

$$
\frac{s_{n, j_{x}}(x) f\left(\frac{j_{x} \pi}{n}\right)}{s_{n, k}(x) f\left(\frac{k \pi}{n}\right)} \geq 4 a_{n} \cdot \frac{m_{f}}{M_{f}}
$$


Because $\lim _{n \rightarrow \infty} \frac{4 a_{n} m_{f}}{\pi M_{f}}=\infty$, it follows that there exists $n_{2} \in \mathbb{N}, n_{2} \geq \max \left\{n_{0}, n_{1}\right\}$ such that $\frac{s_{n, j_{x}}(x) f\left(\frac{j_{x} \pi}{n}\right)}{s_{n, k}(x) f\left(\frac{k \pi}{n}\right)}>1$ for all $x \in[c, d], n \geq n_{2}$ and $k \in\{0,1, \ldots, n\}$, $k<j_{x}-a_{n}\left(\right.$ as $\left.k \notin \stackrel{n}{I}_{n, x}\right)$. In addition, it is important to notice that $n_{2}$ does not depend on $x \in[c, d]$, but of course it depends on $f$.

Subcase $\left.\mathrm{i}_{b}\right)$ It is easy to check that $\left.x \in\left[\left(j_{x}+1 / 2\right) \pi\right) / n,\left(j_{x}+1\right) \pi / n\right]$ implies $\left(n x-\left(j_{x}+1\right) \pi\right) \in[-\pi / 2,0]$. Therefore, reasoning as in case $\mathrm{i}_{a}$ ) (because of $\sin ^{2}$ ) we obtain that for sufficiently large $n$ we have

$$
\frac{s_{n, j_{x}+1}(x) f\left(\frac{\left(j_{x}+1\right) \pi}{n}\right)}{s_{n, k}(x) f\left(\frac{k \pi}{n}\right)}>1, x \in[c, d] .
$$

Summarizing, we conclude that there exists an absolute constant $N_{1} \in \mathbb{N}$ which depends only on $a, b, c, d, f$ such that

$$
\frac{\max \left\{s_{n, j_{x}}(x) f\left(j_{x} \pi / n\right), s_{n, j_{x}+1}(x) f\left(\left(j_{x}+1\right) \pi / n\right)\right\}}{s_{n, k}(x) f(k \pi / n)}>1
$$

for all $x \in[c, d], n \geq N_{1}$ and $k \in\{0,1, \ldots, n\}, k<j_{x}-a_{n}$.

Case ii) The proof is identical with the proof of the above Case i) and therefore we conclude that there exists an absolute constant $N_{2} \in \mathbb{N}$ which depends only on $a, b, c, d, f$ such that

$$
\frac{\max \left\{s_{n, j_{x}}(x) f\left(j_{x} \pi / n\right), s_{n, j_{x}+1}(x) f\left(\left(j_{x}+1\right) \pi / n\right)\right\}}{s_{n, k}(x) f(k \pi / n)}>1
$$

for all $x \in[c, d], n \geq N_{2}$ and $k \in\{0,1, \ldots, n\}, k>j_{x}+a_{n}$.

Analyzing the results obtained in cases i)-ii), it results that for all $x \in[c, d]$, $n \geq N_{0}, N_{0}=\max \left\{N_{1}, N_{2}\right\}$ and $k \in\{0,1, \ldots, n\}$, with $k<j_{x}-a_{n}$ or $k>j_{x}+a_{n}$, we have

$$
\frac{\max \left\{s_{n, j_{x}}(x) f\left(j_{x} \pi / n\right), s_{n, j_{x}+1}(x) f\left(\left(j_{x}+1\right) \pi / n\right)\right\}}{s_{n, k}(x) f(k \pi / n)}>1 .
$$

In conclusion, we obtain our preliminary result, that is

$$
\bigvee_{k=0}^{n} s_{n, k}(x) f\left(\frac{k \pi}{n}\right)=\bigvee_{k \in I_{n, x}} s_{n, k}(x) f\left(\frac{k \pi}{n}\right),
$$

where $I_{n, x}=\left\{k \in\{0,1, \ldots, n\}: j_{x}-a_{n} \leq k \leq j_{x}+a_{n}\right\}$.

Next, let us choose arbitrary $x \in[c, d]$ and $n \in \mathbb{N}$ so that $n \geq N_{0}$. If there exists $k \in I_{n, x}$ such that $k \pi / n \notin[c, d]$ then we distinguish two cases. Either $k \pi / n<c$ or $k \pi / n>d$. In the first case we observe that

$$
0<c-\frac{k \pi}{n} \leq x-\frac{k \pi}{n} \leq \frac{\left(j_{x}+1\right) \pi}{n+1}-\frac{k \pi}{n} \leq \frac{\left(j_{x}+1\right) \pi}{n}-\frac{k \pi}{n} \leq \frac{\left(a_{n}+1\right) \pi}{n} .
$$


Since $\lim _{n \rightarrow \infty} \frac{\left(a_{n}+1\right) \pi}{n}=0$, it results that for sufficiently large $n$ we necessarily have $\frac{\left(a_{n}+1\right) \pi}{n}<c-a$ which clearly implies that $k \pi / n \in[a, c]$. In the same manner, when $k \pi / n>d$, for sufficiently large $n$ we necessarily have $k \pi / n \in[d, b]$.

Summarizing, there exists $\widetilde{N}_{1} \in \mathbb{N}$ independent of any $x \in[c, d]$, such that

$$
\bigvee_{k=0}^{n} s_{n, k}(x) f\left(\frac{k \pi}{n}\right)=\bigvee_{k \in I_{n, x}} s_{n, k}(x) f\left(\frac{k \pi}{n}\right), n \geq \widetilde{N}_{1}
$$

and in addition for any $x \in[c, d], n \geq \widetilde{N}_{1}$ and $k \in I_{n, x}$, we have $k \pi / n \in[a, b]$. Also, it is easy to check that $\widetilde{N}_{1}$ depends only on $a, b, c, d, f$. We thus obtain that

$$
T_{n}^{(M)}(f)(x)=\frac{\bigvee_{k \in I_{n, x}} s_{n, k}(x) f\left(\frac{k \pi}{n}\right)}{\bigvee_{k=0}^{n} s_{n, k}(x)}, n \geq \widetilde{N}_{1}, x \in[c, d]
$$

and in addition for any $x \in[c, d], n \geq \widetilde{N}_{1}$ and $k \in I_{n, x}$, we have $k \pi / n \in[a, b]$.

Reasoning for the function $g$ exactly as in the case of the function $f$, it follows that there exists $\widetilde{N}_{2} \in \mathbb{N}$ which depends only on $a, b, c, d, g$ such that

$$
T_{n}^{(M)}(g)(x)=\frac{\bigvee_{k \in I_{n, x}} s_{n, k}(x) g\left(\frac{k \pi}{n}\right)}{\bigvee_{k=0}^{n} s_{n, k}(x)}, n \geq \widetilde{N}_{2}, x \in[c, d]
$$

and in addition for any $x \in[c, d], n \geq \widetilde{N}_{2}$ and $k \in I_{n, x}$, we have $k \pi / n \in[a, b]$. Taking $\widetilde{n}=\max \left\{\widetilde{N}_{1}, \widetilde{N}_{2}\right\}$, we easily obtain the desired conclusion.

As a consequence of the localization result in Theorem 5.1 we present a local shape preserving property.

Corollary 5.2. Let $f:[0, \pi] \rightarrow \mathbb{R}_{+}$be bounded on $[0, \pi]$ with strictly positive lower bound and suppose that there exists $a, b \in[0, \pi], 0<a<b<\pi$ such that $f$ is constant on $[a, b]$ with the constant value $\alpha>0$. Then for any $c, d \in[a, b]$ with $a<c<d<b$, there exists $\widetilde{n} \in \mathbb{N}$ which depends only on $a, b, c, d$ and $f$ such that $T_{n}^{(M)}(f)(x)=\alpha$ for all $x \in[c, d]$ and $n \in \mathbb{N}, n \geq \widetilde{n}$.

Proof. Let $g:[0,1] \rightarrow \mathbb{R}_{+}$be given by $g(x)=\alpha>0$ for all $x \in[0, \pi]$. Since $f(x)=g(x)$ for all $x \in[a, b]$ and since obviously $T_{n}^{(M)}(g)(x)=\alpha$ for all $x \in[0, \pi]$, by Theorem 5.1 we easily obtain the desired conclusion.

At the end of this section, as a consequence of the localization result in Theorem 5.1 we present a direct result, as follows.

Corollary 5.3. Let $f:[0, \pi] \rightarrow \mathbb{R}_{+}$be bounded on $[0, \pi]$ with the lower bound strictly positive and suppose that there exists $a, b \in[0, \pi], 0<a<b<\pi$ and the constant $C_{0}$ which depends only on $a$ and $b$, such that

$$
|f(x)-f(y)| \leq C_{0}|x-y| \text { for all } x \in[a, b],
$$


that is $\left.f\right|_{[a, b]} \in \operatorname{Lip}[a, b]$. Then, for any $c, d \in[0,1]$ satisfying $a<c<d<b$, we have

$$
\left|T_{n}^{(M)}(f)(x)-f(x)\right| \leq \frac{C}{n} \text { for all } n \in \mathbb{N} \text { and } x \in[c, d],
$$

where the constant $C$ depends only on $f$ and the values $a, b, c, d$.

Proof. Let us define the function $F:[0, \pi] \rightarrow \mathbb{R}$,

$$
F(x)=\left\{\begin{array}{lll}
f(x) & \text { if } & x \in[a, b], \\
f(a) & \text { if } & x \in[0, a], \\
f(b) & \text { if } & x \in[b, \pi] .
\end{array}\right.
$$

The hypothesis imply that $F$ is continuous strictly positive and, according to Theorem 4.4 in [11] it results that

$$
\left|T_{n}^{(M)}(F)(x)-F(x)\right| \leq 4 \omega_{1}\left(F, \frac{1}{n}\right), x \in[0,1], n \in \mathbb{N},
$$

Since by the definition of $F$ we have $\omega_{1}\left(F, \frac{1}{n}\right)_{[0, \pi]} \leq \omega_{1}\left(f, \frac{1}{n}\right)_{[a, b]}$ and since by the relation (8) it easily follows $\omega_{1}\left(f, \frac{1}{n}\right)_{[a, b]} \leq C_{0} / n$, we get

$$
\left|T_{n}^{(M)}(F)(x)-F(x)\right| \leq 4 C_{0} / n, x \in[0, \pi], n \in \mathbb{N},
$$

Now, let us choose arbitrary $c, d \in[a, b]$ such that $a<c<d<b$. Then, by Theorem 5.1 (applicable to $F$ and $f$ ) it results the existence of $\widetilde{n} \in \mathbb{N}$ which depends only on $a, b, c, d, f, F$ such that $T_{n}^{(M)}(F)(x)=T_{n}^{(M)}(f)(x)$ for all $x \in$ $[c, d]$. But since actually the function $F$ depends on the function $f$, it is clear that in fact $\widetilde{n}$ depends only on $a, b, c, d$ and $f$.

Therefore, for arbitrary $x \in[c, d]$ and $n \in \mathbb{N}$ with $n \geq \widetilde{n}$ we obtain

$$
\left|T_{n}^{(M)}(f)(x)-f(x)\right|=\left|T_{n}^{(M)}(F)(x)-F(x)\right| \leq \frac{4 C_{0}}{n},
$$

where $C_{0}$ and $\widetilde{n}$ depend only on $a, b, c, d$ and $f$.

Now, denoting

$$
C_{1}=\max _{1 \leq n<\widetilde{n}}\left\{n \cdot\left\|T_{n}^{(M)}(f)-f\right\|_{[c, d]}\right\}
$$

we finally obtain

$$
\left|T_{n}^{(M)}(f)(x)-f(x)\right| \leq \frac{C}{n}, \text { for all } n \in \mathbb{N}, x \in[c, d],
$$

with $C=\max \left\{C_{0}, C_{1}\right\}$ depending only on $a, b, c, d$ and $f$. This proves the corollary.

Remark. The results in Theorem 5.1 and Corollary 5.2 show the nice property of the truncated max-product operator $T_{n}^{(M)}$ to reproduce locally with great 
accuracy the graph of non-smooth strictly positive, bounded and locally continuous function $f$, with important implications in the signals' representation. For example, if on a subinterval $[c, d]$ the signal $f$ is zero, we may consider there to be constant equal to an arbitrary small $\varepsilon>0$ and then, the above Corollary 5.2 proves that for sufficiently large $n, T_{n}^{(M)}(f)(x)=\varepsilon$ for all $x \in[c, d]$. Note that the truncated linear sampling operator $T_{n}$ does not have these properties.

\section{Localization, Local Inverse and Local Direct Satu- ration Results for the $W_{n}^{(M)}$ Operator}

In this section we present a local inverse saturation result, a strong localization result and a local direct saturation result for the $W_{n}^{(M)}$ operator.

In this sense, let us make the following remarks. Firstly, note that the proof of Theorem 4.1 follows from Lemma 4.4 and the proof of Lemma 4.4 follows from the Lemmas 4.2-4.3 (which are independent of any kind of operator) and Lemmas 2.2-2.3. Since the Lemmas 2.2-2.3 are identical with Lemmas 3.2-3.3 (making abstraction of the kernels), in the case of the truncated max-product operator based on Whittaker-type kernel we easily obtain corresponding results for Theorems 4.1 and 5.1 and for Corollaries 5.2 and 5.3, which are the main results of the previous two sections.

In conclusion, for $W_{n}^{(M)}$ we can state the following local inverse saturation result, localization result and local direct saturation result, respectively.

Theorem 6.1. Let $f:[0, \pi] \rightarrow \mathbb{R}_{+}$and $0<\alpha<\beta<\pi$ be such that $f$ is continuous on $[\alpha, \beta]$. If there exists a constant $M>0$ (independent of $n$ but depending on $f, \alpha$ and $\beta$ ) such that

$$
\left\|W_{n}^{(M)}(f)-f\right\|_{[\alpha, \beta]} \leq M / n, \text { for all } n \in \mathbb{N},
$$

then $\left.f\right|_{[\alpha, \beta]} \in \operatorname{Lip}[\alpha, \beta]$, that is $f$ is a Lipschitz function on $[\alpha, \beta]$.

Theorem 6.2. Let $f, g:[0, \pi] \rightarrow \mathbb{R}_{+}$be both bounded on $[0, \pi]$ with strictly positive lower bounds and suppose that there exists $a, b \in[0, \pi], 0<a<b<\pi$ such that $f(x)=g(x)$ for all $x \in[a, b]$. Then for all $c, d \in[a, b]$ satisfying $a<c<d<b$ there exists $\widetilde{n} \in \mathbb{N}$ which depends only on $f, g, a, b, c, d$ such that $W_{n}^{(M)}(f)(x)=W_{n}^{(M)}(g)(x)$ for all $x \in[c, d]$ and $n \in \mathbb{N}, n \geq \widetilde{n}$.

Corollary 6.3. Let $f:[0, \pi] \rightarrow \mathbb{R}_{+}$be bounded on $[0, \pi]$ with strictly positive lower bound and suppose that there exists $a, b \in[0, \pi], 0<a<b<\pi$ and the constant $C_{0}$ which depends only on $a$ and $b$, such that

$$
|(f)(x)-f(y)| \leq C_{0}|x-y| \text { for all } x \in[a, b],
$$

that is $\left.f\right|_{[a, b]} \in \operatorname{Lip}([a, b])$. Then, for any $c, d \in[0,1]$ satisfying $a<c<d<b$, we have

$$
\left|W_{n}^{(M)}(f)(x)-f(x)\right| \leq \frac{C}{n} \text { for all } n \in \mathbb{N} \text { and } x \in[c, d],
$$


where the constant $C$ depends only on $f$ and the values $a, b, c, d$.

Corollary 6.4. Let $f:[0, \pi] \rightarrow \mathbb{R}_{+}$be bounded on $[0, \pi]$ with strictly positive lower bound and suppose that there exists $a, b \in[0, \pi], 0<a<b<\pi$ such that $f$ is constant on $[a, b]$ with the constant value $\alpha>0$. Then for any $c, d \in[a, b]$ with $a<c<d<b$, there exists $\widetilde{n} \in \mathbb{N}$ which depends only on $a, b, c, d$ and $f$ such that $W_{n}^{(M)}(f)(x)=\alpha$ for all $x \in[c, d]$ and $n \in \mathbb{N}, n \geq \widetilde{n}$.

Remarks. 1) Similar to the case of the operator $T_{n}^{(M)}$, the results in the Theorem 6.2 and Corollary 6.4 show the nice properties of the truncated maxproduct operator $W_{n}^{(M)}$ to reproduce locally with great accuracy the graph of a non-smooth strictly positive, bounded and locally continuous function $f$, with important potential applications in the signals' representation. Note that the truncated linear sampling operator $W_{n}$ does not have these properties.

2) Although the max-product operators $W_{n}^{(M)}$ and $T_{n}^{(M)}$ studied by this paper are well-defined only for bounded functions with values in $\mathbb{R}_{+}$, they can easily be used to generate max-product type operators for the approximation of functions of arbitrary sign $f:[0, \pi] \rightarrow \mathbb{R}$, as follows : if $a<$ $\min \{f(x) ; x \in[0, \pi]\}$, then the new operators $P_{n}^{(M)}(f)(x)=W_{n}^{(M)}(f-a)(x)+a$ and $Q_{n}^{(M)}(f)(x)=T_{n}^{(M)}(f-a)(x)+a$, approximate $f$ with the same Jacksontype order $\omega_{1}(f ; 1 / n)_{[0, \pi]}$ and keep the interpolation properties $P_{n}^{(M)}(f)(j \pi / n)=$ $Q_{n}^{(M)}(j \pi / n)=f(j \pi / n), j=0,1, \ldots, n$.

\section{ACKNOWLEDGEMENT}

The work of both authors was supported by a grant of the Romanian National Authority for Scientific Research, CNCS-UEFISCDI, project number PN-II-IDPCE-2011-3-0861.

\section{References}

[1] C. Bardaro, P.L. Butzer, R.L. Stens, G. Vinti, Approximation error of the Whittaker cardinal series in terms of an averaged modulus of smoothness covering discontinuous signals, J. Math. Anal. Appl. 316, 269-306, 2006.

[2] C. Bardaro, P.L. Butzer, R.L. Stens, G. Vinti, Kantorovich-type generalized sampling series in the setting of Orlicz spaces, Sampl. Theory Signal Image Process., 6(1), 19-52, 2007.

[3] C. Bardaro, P.L. Butzer, R.L. Stens, G. Vinti, Prediction by samples from the past with error estimates covering discontinuous signals, IEEE Trans. on Information Theory, 56(1), 614-633, 2010. 
[4] B. Bede, L. Coroianu, S.G. Gal, Approximation and shape preserving properties of the Bernstein operator of max-product kind, Intern. J. Math. Math. Sci., vol. 2009, Article ID 590589, 26 pages, 2009. DOI:10.1155/2009/590589.

[5] E. Borel, Sur l'interpolation, C.R. Acad. Sci. Paris, 124, 673-676, 1897.

[6] P.L. Butzer, A survey of the Whittaker-Shannon sampling theorem and some of its extensions, J. Math. Res. Expos., 3, 185-212, 1983.

[7] P.L. Butzer, W. Engels, S. Ries, R.L. Stens, The Shannon sampling series and the reconstruction of signals in terms of linear, quadratic and cubic splines, SIAM Journal on Applied Mathematics, 46(2), 299-323, 1986.

[8] P.L. Butzer, W. Splettstößer, R.L. Stens, The sampling theorems and linear prediction in signal analysis, Jahresber. Deutsch. Math-Verein, 90, 1-70, 1988.

[9] P.L. Butzer, R.L. Stens, The Poisson summation formula, Whittaker's cardinal series and approximate integration, in : Proc. Second Edmonton Conference on Approximation Theory, Canadian Mathematical Society, 3, 1936, 1983.

[10] L. Coroianu, S.G. Gal, Approximation by nonlinear generalized sampling operators of max-product kind, Sampl. Theory Signal Image Process., 9(13), 59-75, 2010.

[11] L. Coroianu, S.G. Gal, Approximation by max-product sampling operators based on sinc-type kernels, Sampl. Theory Signal Image Process., 10(3), 211-230, 2011.

[12] S. G. Gal, Shape-Preserving Approximation by Real and Complex Polynomials, Birkhäuser, Boston-Basel-Berlin, 2008.

[13] A. Kivinukk, G. Tamberg, Interpolating generalized Shannon sampling operators, their norms and approximation properties, Sampl. Theory Signal Image Process., 8(1), 77-95, 2009.

[14] A. Kivinukk, G. Tamberg, On approximation properties of sampling operators by dilated kernels, 8th Intern. Conf. on Sampling Theory and Applications, SampTA'09, Marseille, May 18-22, 2009, Poster sessions, electronic access at www.latp.univ-mrs.fr/SAMPTA09/FinalSubmissions/187.pdf

[15] G. Plana, Sur une nouvelle expression analytique des nombers Bernoulliens, Academia di Torino, 25, 403-418, 1820. 
[16] V. P. Sklyarov, On the best uniform sinc-approximation on a finite interval, East J. Approx., 14(2), 183-192, 2008.

[17] R. L. Stens, Approximation of functions by Whittaker's cardinal series, in : General Inequalities 4, Proc. Conference, Oberwolfach, Germany, May 1983, W. Walter ed., ISNM 71, Birkhauser Verlag, Basel, pp. 137-149, 1984.

[18] M. Theis, Über eine Interpolationsformel von de la Vallée-Poussin, Math. Z., 3, 93-113, 1919.

[19] A.Yu. Trynin, A criterion for the uniform convergence of sinc-approximation on a segment, Russian Mathematics (Iz. VUZ), 52(6), 58-69, 2008.

[20] E.T. Whittaker, On the functions which are represented by expansions of the interpolation theory, Proc. Roy. Soc. Edinburgh, 35, 181-194, 1915. 